categories: managed, labour and repetitive motion types through descriptive statistics and two way ANOVA. Indicate the relationship between the two sets of 8 weeks of physical health intervention program.

Results Stress and lack of sleep were the sources of fatigue. The degree of discomfort in neck and shoulder and low back were related to the level of fatigue. There was a significant decrease in lower limb and back muscle stiffness and an increase in muscle elasticity measurements after physical health promotion program. Managed workers showed muscle tension is greater than the vertical type of labour.

Conclusions A well planned physical health program specifically designed the needs for the workforces can effectively change the perception of fatigue and reduce the level of muscle stiffness. Such promotion model can be further utilised in other occupational worksites.

\section{PREDICTING PHYSICIAN'S DUTY STRESS BY PARASYMPATHETIC NERVOUS FUNCTION (ALSO TO BE CONSIDERED FOR MINI-SYMPOSIUM: EARLY DETECTION AND MANAGEMENT OF WORKERS UNDER STRESS)}

${ }^{1,2}{ }^{2}$ Hsiu-Hao Lee, ${ }^{1}$ Bing-Yu Chen, ${ }^{2}$ Shih-Hsiang Lo, ${ }^{1}$ Pau-Chung Chen, 1,3Yue-Liang Guo. ${ }^{1}$ Institution of Occupational Medicine and Industrial Hygiene, National Taiwan University College of Public Health, Taipei, Taiwan; ${ }^{2}$ Department of Internal Medicine, Taipei City Hospital, Zhongxing Branch, Taipei, Taiwan; ${ }^{3}$ Department of Environmental and Occupational Medicine, National Taiwan University College of Medicine and NTU Hospital, Taipei, Taiwan

\subsection{6/oemed-2014-102362.290}

Objectives Early stress markers are useful in detecting workers with occupational stress. The aim of this study was to determine whether heart rate variability was associated with physicians' duty loading, and also a good predictor for stress markers.

Method An observational study on physicians with variable duty loading was conducted in a secondary referral medical centre in northern Taiwan in 2012. For every participant, 24-hr electrocardiography (EKG) and hourly blood pressure were obtained during three test days, i.e., regular-duty (only day shift), moderate-duty (day and night-shift with moderate number of patients cared), and high-duty days (day and night-shift with higher number of patients cared). Blood samples for stress markers were obtained at 8 am on the test day, and 8 am on the second morning.

Results A total of 12 staff physicians satisfactorily completed the study. The number of patients covered at night shift was $0,92 \pm$ 8 , and $187 \pm 9$, for regular-, moderate-, and high-duty nights, respectively. Total phone calls, urgent procedures, new patients admitted, critical patients cared and times of awakenings were significantly higher as the duty loads increased. The parasympathetic indicator derived from continuous EKG, high frequency normalised unit (HFnu), was negatively related to loading of total patient cared $(\mathrm{P}<0.0001)$. Reduced HFnu predicted elevated night systolic blood pressure $(\mathrm{P}=0.016)$ and serum uric acid $(\mathrm{P}=0.024)$, and $24 \mathrm{~h}$ urine vanillylmandelic acid $(\mathrm{P}=$ 0.0045), dopamine $(\mathrm{P}=0.011)$, and norepinephrine $(\mathrm{P}=$ 0.027).

Conclusions HFnu derived from heart rate variability measurement may predict several important stress markers during nightshift duties.

\section{DETERMINANTS OF OCCUPATIONAL HYGIENE EXPERT JUDGMENT ACCURACY}

${ }^{1}$ Mohammad Javad Zare Sakhvidi, ${ }^{1}$ Hamideh Mihanpoor, ${ }^{1}$ Abolfazl Barkhordari, ${ }^{2}$ Mehrdad Mostachaci. 'Department of Occupational Health, Faculty of Health, Shahid Sadoughi University of Medical Sciences, Yazd, Iran; ${ }^{2}$ Department of Occupational Medicine, Faculty of Medicine, Shahid Sadoughi University of Medical Sciences, Yazd, Iran

\subsection{6/oemed-2014-102362.291}

Objectives A cross sectional study was performed to determine the applicability and accuracy of expert judgment in occupational exposure assessment. The roles of educational session and determining factors were also realised.

Method Thirteen occupational hygienists were divided into two groups based on their field experience. They asked to evaluate exposure intensity in seven operating units in a tile factory before and after exposure training session. Participant's judgments were compared to actual air sampling data in the factory; and relative errors were calculated. Inter-class correlation coefficients were calculated and relative errors compared according to participants characteristics. Stepwise regressions were performed to investigate the defining variables.

Results In all situations there were almost perfect agreement (ICC >0.80) among raters. Correlations between estimated mean exposure and relative percentage error of participants before and after training were significant at 0.01 (correlation coefficients were -0.462 and -0.443 respectively). Results showed that actual concentration and experience resulted in $22.4 \%$ prediction variance for expert error as an independent variable.

Conclusions Correctness of exposure ratings by hygienists was susceptible to error from several sources. It seems that experienced subjects had better ability to predict the exposures. In general, in lower concentrations, the rating error increased significantly. Leading causes of judgment error should be taken into account in epidemiological exposure assessment studies.

\section{OCCUPATIONAL EXPOSURE AND STROKE - A CRITICAL REVIEW OF CHEMICAL AND PHYSICAL EXPOSURES}

1,2 $\mathrm{Per}$ Gustavsson, ${ }^{3}$ Kristina Jakobsson. ${ }^{1}$ Institute of Environmental Medicine, Karolinska Institutet, Stockholm, Sweden; ${ }^{2}$ Centre for Occupational and Environmental Medicine, Stockholm County Council, Stockholm, Sweden; ${ }^{3}$ Division of Occupational and Environmental Medicine, Lund University, Stockholm, Sweden

\subsection{6/oemed-2014-102362.292}

Objectives Stroke is the third most common cause of death in developed countries, exceeded only by coronary heart disease and cancer. There is substantial scientific literature on the association between occupational exposures and coronary heart disease, but much less is known about stroke. This systematic critical review was performed to assess the strength of evidence for causal associations between chemical and physical occupational exposures and stroke.

Method Literature on stroke incidence or mortality and occupational factors published up to 2012 was identified from Medline and Scopus. The 4471 abstracts were evaluated independently by two reviewers. 29 studies relevant to chemical and physical exposures were identified; ionising irradiation (7 studies); carbon disulfide (4), dynamite (3), motor exhaust (7) and other combustions products (8). The evidence for an association was assessed according to defined criteria as strong, moderate, limited, or insufficient. 